\title{
Seamless Graphene-Seal-Wrap as Removable Protective Cover for Two-Dimensional Materials
}

Kailun Xia ${ }^{a}$, Muqiang Jian ${ }^{a}$, Chunya Wang ${ }^{a}$, Zhe Yin ${ }^{a}$, Mingchao Zhang ${ }^{a}$, Huimin Wang, Yang $W u^{b}$ and Yingying Zhang ${ }^{a^{*}}$

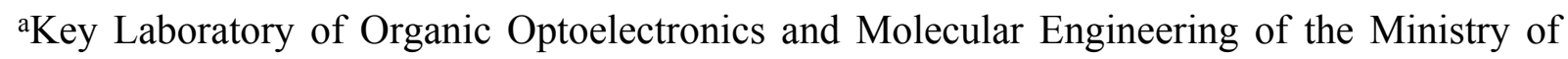
Education, Department of Chemistry, Tsinghua University, Beijing 100084, PR China

bDepartment of Mechanical Engineering, Tsinghua University, Beijing 100084, PR China

*Address correspondence to yingyingzhang@tsinghua.edu.cn

This supporting file includes:

Experimental Section

Supporting Figures S1 to S11 


\section{Experimental Section}

Growth of the high-quality graphene film. The graphene film was grown on $\mathrm{Cu}$ foil $(25 \mu \mathrm{m}$ thick, Alfa Aesar Inc) by CVD. Firstly, the $\mathrm{Cu}$ foil was cleaned in $\mathrm{FeCl}_{3} / \mathrm{HCl}(0.5 \mathrm{~mol} / \mathrm{L})$ solution for $15 \mathrm{~s}$ to remove the surface oxides and then washed with acetone, isopropanol and deionized (DI) water for 15 min, respectively. After being dried with a nitrogen gun, the $\mathrm{Cu}$ foil was put into the CVD chamber and heated to $1065^{\circ} \mathrm{C}$ with the flow of $\mathrm{Ar}(500 \mathrm{sccm}$, 99.999\% purity) and hydrogen $\left(\mathrm{H}_{2}, 100 \mathrm{sccm}, 99.999 \%\right.$ purity) in $60 \mathrm{~min}$. This system was kept for 5 hours. The growth was carried out with the introduction of methane $\left(\mathrm{CH}_{4}, 0.2\right.$ sccm, $99.999 \%$ purity) for $50 \mathrm{~min}$. The growth was terminated by stopping the $\mathrm{CH}_{4}$ flow and cooling down in the flow of $\mathrm{Ar}$ and $\mathrm{H}_{2}$.

Fabrication of GSW. (1) Spin coat a layer of PMMA on the graphene/Cu sample (3000 $\mathrm{r} / \mathrm{min}$ for $30 \mathrm{~s}$ ). (2) Bake the sample in a thermal drying oven at $70{ }^{\circ} \mathrm{C}$ for $30 \mathrm{~min}$. (3) Cover a HRT on the PMMA/graphene/Cu surface and remove the residual small air bubbles between the PMMA and the HRT in a vacuum chamber (with a vacuum of $10^{-5} \mathrm{~Pa}$ ). (4) Put the sample into $\mathrm{FeCl}_{3} / \mathrm{HCl}$ solution $(1 \mathrm{~mol} / \mathrm{L}$ ) to etch the $\mathrm{Cu}$ foil. (5) Clean the sample with DI water for three times and dry with a nitrogen gun.

Seal/unseal of BP using the GSW. The sealing process is as the following. (1) Cover the BP sample with GSW and place it in a vacuum chamber $\left(10^{-5} \mathrm{~Pa}\right)$ to remove the residual air between the GSW and BP. (2) Heat to $120^{\circ} \mathrm{C}$ and maintain for 10 min to release the HRT film. (3) Heat the sample at $350{ }^{\circ} \mathrm{C}$ or dip the sample into acetone to remove the PMMA layer. The unseal process is as the following. (1) Cover the surface of graphene/BP sample with a HRT film and place it in the vacuum chamber (with a vacuum of $10^{-5} \mathrm{~Pa}$ ) to remove the residual air between the film and graphene. (2) Peel off the HRT/graphene film directly. 
Characterization. The morphologies of the BP sheets were characterized by an optical microscope (LEICA DM2500 M), and an atomic force microscopy (AFM) (NanoScope V, Veeco). The Raman spectrum was obtained using a laser excitation wavelength of $532 \mathrm{~nm}$.

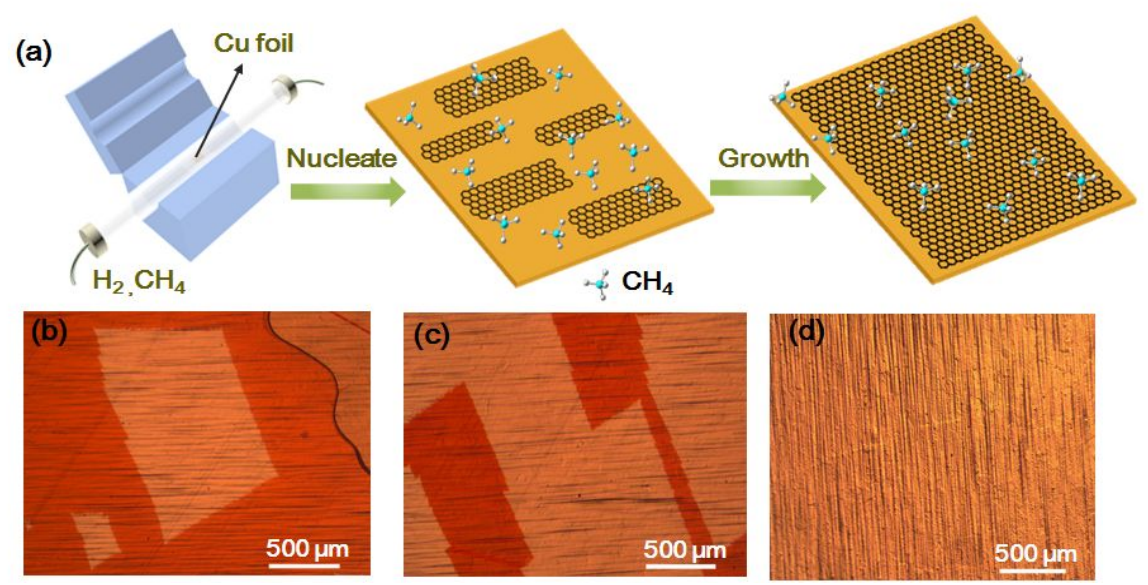

Figure S1. CVD growth of high quality graphene film. (a) Schematic diagram showing the CVD growth of graphene film consisted of aligned graphene domains. (b) Optical image of a graphene domain. (c) Optical image of a graphene sample that has not been formed into a complete film. (d) Optical image of a seamless graphene film.

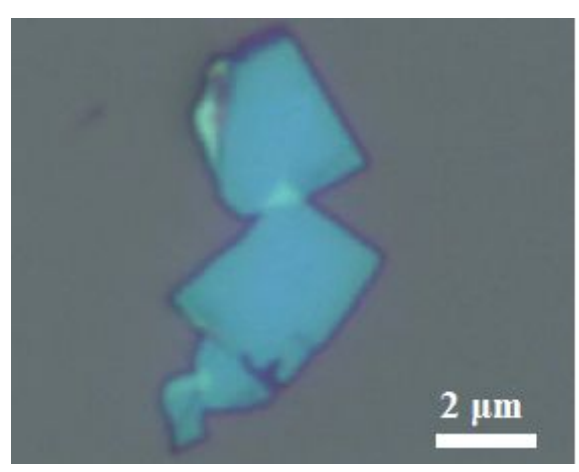

Figure S2. Optical image of a graphene/BP sample. 


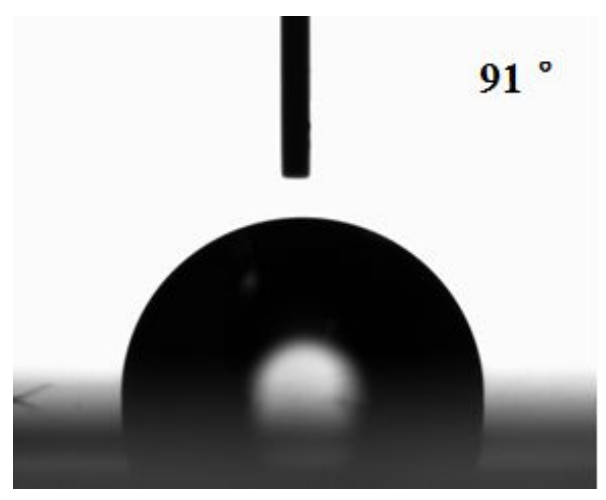

Figure S3. The water contact angle of a graphene/BP sample.

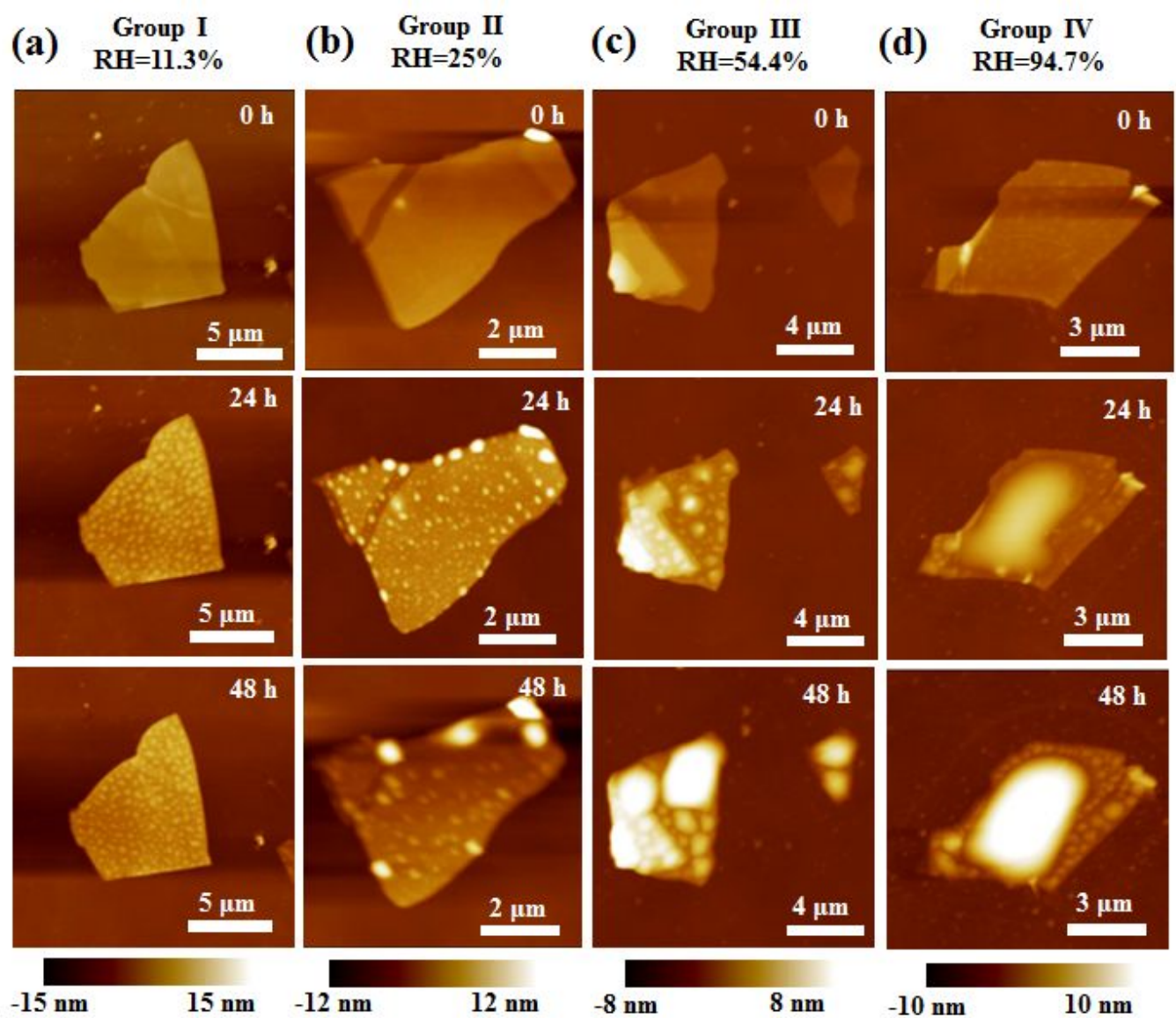

Figure S4. AFM images showing gradual deterioration of bare BP sheets at different humidity. (a) RH of $11.3 \%$. (b) RH of $25 \%$. (c) RH of $54.4 \%$. (d) RH of $94.7 \%$. 


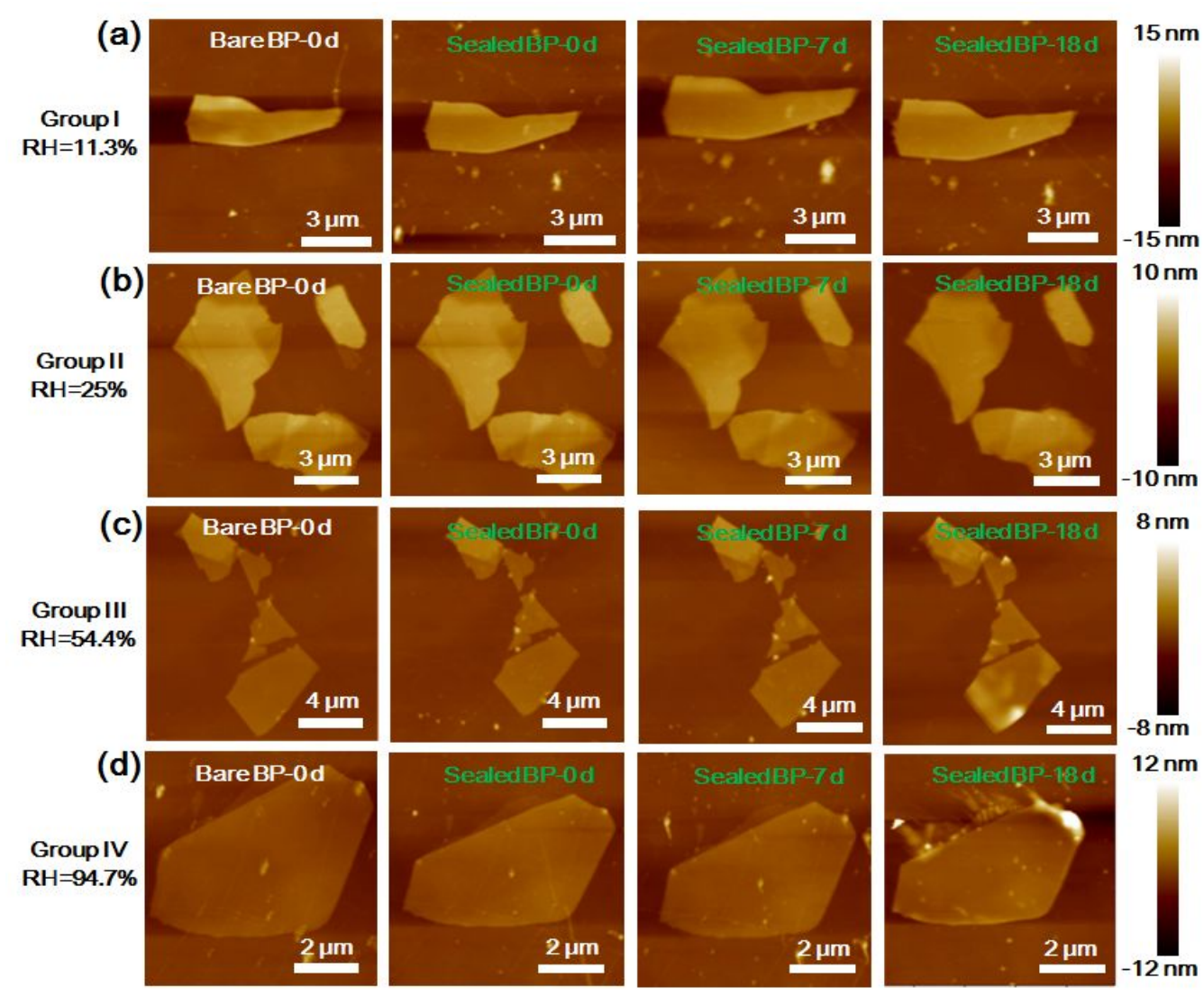

Figure S5. AFM images showing the graphene protected BP sheets at different humidity after being storage for different duration. (a) $\mathrm{RH}$ of $11.3 \%$. (b) $\mathrm{RH}$ of $25 \%$. (c) $\mathrm{RH}$ of $54.4 \%$. (d) RH of $94.7 \%$.
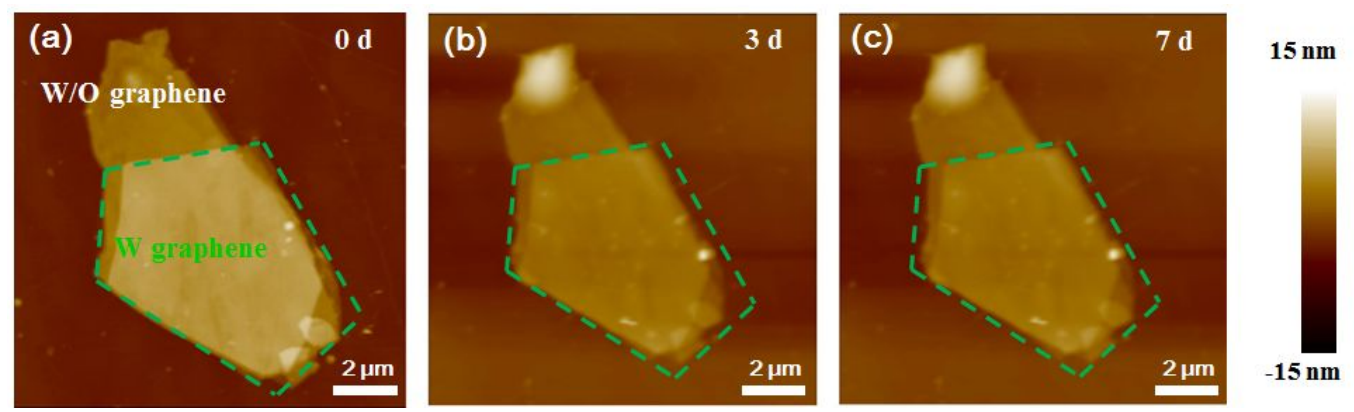

Figure S6. AFM images showing the evolution of a BP sheet partially covered with graphene. 


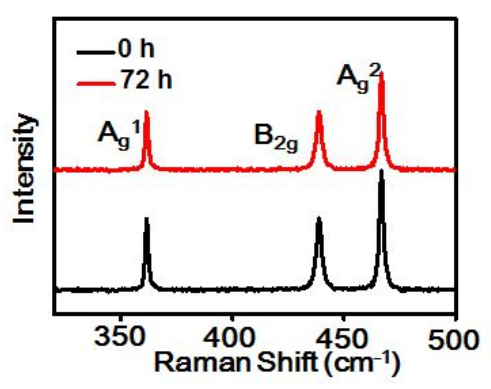

Figure S7. Raman results of graphene/BP sample after being exposed to air for $0 \mathrm{~h}$ and $72 \mathrm{~h}$. 